\title{
THREE QUBIT SEPARABLE STATES OF LENGTH TEN WITH UNIQUE DECOMPOSITIONS
}

\author{
SEUNG-HYEOK KYE
}

\begin{abstract}
We construct one parameter families of three qubit separable states with length ten, which is strictly greater than the whole dimension eight. These states are located on the boundary of the convex set of all separable states, but they are in the interior of the convex set of all states with positive partial transposes. They are also decomposed into the convex sum of ten pure product states in a unique way.
\end{abstract}

\section{INTRODUCTION}

Entanglement is one of the key notions in the current quantum information theory, and it is an important research topic to distinguish entanglement from separability. Recall that a multi-partite state is said to be separable if it is a convex combination of pure product states, and entangled if it is not separable. The length of separable states is one of the notions to understand the convex structures of the convex set $\mathcal{S}$ consisting of all separable states, as it was considered by several authors [10, 18, 23, 24, 25, 26, 28] in the early stages of quantum information theory.

The length $\ell(\varrho)$ of a separable state $\varrho$ is defined by the smallest number of pure product states with which $\varrho$ may be expressed as a convex combination. Physically, it represents the minimal physical effort to implement the state. It is clear that the length $\ell(\varrho)$ is bigger than or equal to the rank of $\varrho$. It was known in [10] that the length may be strictly bigger than the rank. On the other hand, it was shown $[3$ for $2 \otimes 3$ bi-partite case that the length $\ell(\varrho)$ of a separable state $\varrho$ coincides with the maximum of the ranks of $\varrho$ and its partial transpose $\varrho^{\Gamma}$. This is not the case for the $3 \otimes 3$ system, by examples 14 of separable states $\varrho$ with $\ell(\varrho)=6$ but $\operatorname{rank} \varrho=\operatorname{rank} \varrho^{\Gamma}=5$.

The length cannot exceed the whole affine dimension of the system by the classical Caratéodory theorem, as it was observed in [18]. One natural question is to find the possible maximum of lengths. It was known 24 that $\ell(\varrho) \leq 4$ for every $2 \otimes 2$ separable state $\varrho$. The above mentioned result [3] on $2 \otimes 3$ separable states tells us $\ell(\varrho) \leq 6$ for those separable states. On the other hand, it was shown in [4] that the length of an $m \otimes n$ state may exceeds $m n$ whenever $(m-2)(n-2)>1$, or equivalently $\max \{m, n\} \geq 4$ and $\min \{m, n\} \geq 3$. In the $3 \otimes 3$ and $2 \otimes 4$ systems, examples of separable states of length 10 were found in [12, 14] and [13], respectively, and examples

1991 Mathematics Subject Classification. 81P15, 15A30, 46L07.

Key words and phrases. lengths of separable states, three qubit states, unique decomposition, positive multi-linear maps, dual faces.

partially supported by NRF-2017R1A2B4006655. 
in [14] have been analyzed in [6]. We refer to [7, 8] for further results on the related topics.

In this paper, we consider the three qubit system, the simplest multi-partite case. A result in [4] tells us that there must exist a three qubit separable state of length ten. The main purpose of this paper is to construct explicit examples of such states. Lengths of some three qubit separable states have been calculated in [17] but do not exceed eight, even though some of them exceed the maximum ranks of partial transposes. The states we constructed turn out to be boundary separable states of full ranks in the sense of [6], that is, it is on the boundary of the convex set $\mathcal{S}$, and all the partial transposes have full ranks. Recall that all the partial transposes of a state have full ranks if and only if it is in the interior of the convex set $\mathcal{T}$ of all PPT states.

The main idea is to use the duality [21] between $n$-partite separable states and positive multi-linear maps. For our purpose, we consider the positive bi-linear maps in $2 \times 2$ matrices which have been constructed in [21, 22], and mix variants of them to get positive bi-linear maps whose dual faces have exactly ten pure product states. The positive maps constructed have the full spanning property, and so, the interior of the dual faces are located in the interior of the convex cone of all PPT states. See [21]. Furthermore, the faces are affinely isomorphic to the 9-dimensional simplex with ten extreme points, and so any interior points of the faces are separable states of length ten and are decomposed into the sum of pure product states in a unique way.

Separable states with unique decompositions also have been studied by several authors [1, 2, 15, 9, 13, 14, 19]. The results tell us that separable states generically have unique decompositions if the lengths are sufficiently small. In the multi-partite case of $d_{1} \otimes \cdots \otimes d_{n}$ system, it was shown in [15] that generic choices of $k$ product vectors give rise to separable states with unique decompositions whenever $k \leq \sum_{i=1}^{n}\left(d_{i}-1\right)$, by taking convex sums of corresponding pure product states. In the three qubit case, generic choices of four product vectors still give rise to separable states with unique decompositions. We know [20, 27] that generic five dimensional subspaces of $\mathbb{C}^{2} \otimes$ $\mathbb{C}^{2} \otimes \mathbb{C}^{2}$ have six product vectors. It was also shown [15] that they make three qubit separable states of length six with unique decompositions. Our construction gives examples of three qubit separable states of length ten with unique decompositions.

We collect in the next section basic material about the map in [21], and construct in Section 3 the positive bi-linear map we are looking for.

The author is grateful to Kyung Hoon Han for valuable comments and discussion.

\section{BOUNDARY SEPARABLE STATES WITH FULL RANKS}

For a given multi-linear map $\phi$ from $M_{d_{1}} \times \cdots \times M_{d_{n-1}}$ into $M_{d_{n}}$, we associate the Choi matrix $W_{\phi}$ in $M_{d_{1}} \otimes \cdots \otimes M_{d_{n-1}} \otimes M_{d_{n}}$ by

$$
W_{\phi}=\sum_{i_{1}, j_{1}, \ldots, i_{n-1}, j_{n-1}}\left|i_{1}\right\rangle\left\langle j_{1}|\otimes \cdots \otimes| i_{n-1}\right\rangle\left\langle j_{n-1}\right| \otimes \phi\left(\left|i_{1}\right\rangle\left\langle j_{1}|, \cdots,| i_{n-1}\right\rangle\left\langle j_{n-1}\right|\right),
$$


and define the bi-linear pairing

$$
\langle\varrho, \phi\rangle=\operatorname{Tr}\left(\varrho W_{\phi}^{\mathrm{t}}\right)
$$

for an $(n-1)$-linear map $\phi$ and an $n$-partite state $\varrho$, where $W_{\phi}^{\mathrm{t}}$ denotes the transpose of $W_{\phi}$. We recall that an $(n-1)$-linear map is positive if $\phi\left(x_{1}, \ldots, x_{n-1}\right)$ is positive (semi-definite) whenever all of $x_{1}, \ldots, x_{n-1}$ are positive. It was shown in [21] that $\varrho$ is separable if and only if $\langle\varrho, \phi\rangle \geq 0$ for every positive multi-linear map $\phi$. This tells us that every entanglement witness must be the Choi matrix $W_{\phi}$ of a positive multi-linear map $\phi$.

We have constructed in [21] the positive bilinear map $\phi: M_{2} \times M_{2} \rightarrow M_{2}$ which sends $\left(\left[x_{i j}\right],\left[y_{i j}\right]\right) \in M_{2} \times M_{2}$ to

$$
\left(\begin{array}{cc}
s x_{22} y_{11} & x_{12} y_{12}-x_{12} y_{21}+x_{21} y_{12}+x_{21} y_{21} \\
x_{12} y_{12}+x_{12} y_{21}-x_{21} y_{12}+x_{21} y_{21} & t x_{11} y_{22}
\end{array}\right) \in M_{2},
$$

where $s, t$ are positive numbers with $s t=8$. See also [22] for the motivation of construction. We use the parameter $u>0$ to express the the corresponding entanglement witness, or equivalently the corresponding Choi matrix:

$$
W=\left(\begin{array}{cccccccc}
\cdot & \cdot & \cdot & \cdot & \cdot & \cdot & \cdot & 1 \\
\cdot & \cdot & \cdot & \cdot & \cdot & \cdot & 1 & \cdot \\
\cdot & \cdot & \cdot & \cdot & \cdot & -1 & \cdot & \cdot \\
\cdot & \cdot & \cdot & \sqrt{8} u^{-1} & 1 & \cdot & \cdot & \cdot \\
\cdot & \cdot & \cdot & 1 & \sqrt{8} u & \cdot & \cdot & \cdot \\
\cdot & \cdot & -1 & \cdot & \cdot & \cdot & \cdot & \cdot \\
\cdot & 1 & \cdot & \cdot & \cdot & \cdot & \cdot & \cdot \\
1 & \cdot & \cdot & \cdot & \cdot & \cdot & \cdot & \cdot
\end{array}\right)
$$

with respect to the lexicographic order $000,001,010,011,100,101,110,111$. It was shown [21] that the map $\phi$ is indecomposable positive bi-linear map. In fact, $\phi$ has the full spanning property which implies indecomposability. See also [16] for indecomposability. More recently, the author showed [22] that the map $\phi$ generates an exposed extreme ray of the cone of all positive bi-linear maps between $2 \times 2$ matrix algebras.

We note that all the entries of $W$ are zero except for diagonal and anti-diagonals. Such matrices are called $\mathrm{X}$-shaped, and are of the form

$$
X(a, b, c)=\left(\begin{array}{ccccccccc}
a_{1} & & & & & & & c_{1} \\
& a_{2} & & & & & c_{2} & \\
& & a_{3} & & & c_{3} & & \\
& & & a_{4} & c_{4} & & & \\
& & & \bar{c}_{4} & b_{4} & & & \\
& & \bar{c}_{3} & & & b_{3} & & \\
& \bar{c}_{2} & & & & & b_{2} & \\
\bar{c}_{1} & & & & & & & b_{1}
\end{array}\right),
$$

for vectors $a, b \in \mathbb{R}^{4}$ and $c \in \mathbb{C}^{4}$. Many important three qubit states arise in this form. For example, Greenberger-Horne-Zeilinger diagonal states are $\mathbf{X}$-shaped, and 
$\varrho=X(a, b, c)$ is GHZ diagonal if and only if $a=b$ and $c \in \mathbb{R}^{4}$. With this notation, we note that the above entanglement witnesses $W$ is given by

$$
W=X\left(\left(0,0,0, \sqrt{8} u^{-1}\right),(0,0,0, \sqrt{8} u),(1,1,-1,1)\right) .
$$

We consider the dual face $W^{\prime}$ of $W$, which consists of all separable states $\varrho$ such that $\langle W, \varrho\rangle=0$. In order to understand the structures of the dual face, it is desirable to find all the extreme points of the face, that is, pure product states $\varrho=|\xi\rangle\langle\xi|$ satisfying the relation $\langle W, \varrho\rangle=0$. We have found in [22] all such product vectors $|\xi\rangle$ 's. Note that such a product vector $|\xi\rangle$ satisfies the relation

$$
\langle\bar{\xi}|W| \bar{\xi}\rangle=\langle W, \mid \xi\rangle\langle\xi \mid\rangle=0
$$

In this case, we say that the witness $W$ kills the product vector $|\xi\rangle$, and denote by $P_{W}$ the set of all product vectors killed by $W$ :

$$
P_{W}:=\{|\xi\rangle=|x\rangle \otimes|y\rangle \otimes|z\rangle:\langle W, \mid \xi\rangle\langle\xi \mid\rangle=0\}
$$

Our discussion tells us that there is a one-to-one correspondence between $P_{W}$ and the set of all extreme points of the dual face $W^{\prime}$ of $W$.

We denote by $P_{W}^{\mathrm{z}}$ the set of all product vectors in $P_{W}$ which has zero entries, and by $P_{W}^{\mathrm{n}}$ the complement $P_{W} \backslash P_{W}^{\mathrm{z}}$. For $i, j=0,1$, we denote by $E_{\square i j}$ the set of all three qubit product vectors of the form $|x\rangle \otimes|i\rangle \otimes|j\rangle$ with $|x\rangle \in \mathbb{C}^{2}$. We also define $E_{i \square j}$ and $E_{i j \square}$ similarly:

$$
\begin{aligned}
& E_{\square i j}=\left\{|x\rangle \otimes|i\rangle \otimes|j\rangle:|x\rangle \in \mathbb{C}^{2}\right\}, \\
& E_{i \square j}=\left\{|i\rangle \otimes|x\rangle \otimes|j\rangle:|x\rangle \in \mathbb{C}^{2}\right\}, \\
& E_{i j \square}=\left\{|i\rangle \otimes|j\rangle \otimes|x\rangle:|x\rangle \in \mathbb{C}^{2}\right\} .
\end{aligned}
$$

Note that all the above sets are parameterized by the two dimensional sphere, up to scalar multiplications. It is easy to see that the set $P_{W}^{\mathrm{z}}$ of product vectors in $P_{W}$ with zero entries is given by

$$
P_{W}^{\mathrm{z}}=E_{\square 01} \cup E_{\square 10} \cup E_{0 \square 0} \cup E_{1 \square 1} \cup E_{00 \square} \cup E_{11 \square} .
$$

We proceed to look for product vectors belonging to $P_{W}^{\mathrm{n}}$. For a given triplet $\mathbf{p}=$ $(p, q, r) \in \mathbb{R}_{+}^{3}$ of positive numbers and $\Lambda=(\alpha, \beta, \gamma) \in \mathbb{T}^{3}$, we define the three qubit product vector $|\eta(\mathbf{p}, \Lambda)\rangle$ by

$$
|\eta(\mathbf{p}, \Lambda)\rangle=(p q r)^{-\frac{1}{2}}(p, \alpha)^{\mathrm{t}} \otimes(q, \beta)^{\mathrm{t}} \otimes(r, \gamma)^{\mathrm{t}} \in \mathbb{C}^{2} \otimes \mathbb{C}^{2} \otimes \mathbb{C}^{2} .
$$

Denote by $\omega=e^{\mathrm{i} \frac{\pi}{4}}$ the eighth root of unity, and take eight $\Lambda$ 's in $\mathbb{T}^{3}$ as follows:

$$
\begin{aligned}
& \Lambda_{1}=\left(+\omega^{3},+\omega^{1},+\omega^{7}\right), \\
& \Lambda_{2}=\left(+\omega^{3},-\omega^{1},-\omega^{7}\right), \\
& \Lambda_{3}=\left(-\omega^{3},+\omega^{1},-\omega^{7}\right), \\
& \Lambda_{4}=\left(-\omega^{3},-\omega^{1},+\omega^{7}\right), \\
& \Lambda_{j}=\bar{\Lambda}_{j-4}, \quad j=5,6,7,8 .
\end{aligned}
$$


It was shown in [22] that a product vector without zero entries belongs to $P_{W}$ if and only if it is of the form

$$
\left|\eta_{j}(\mathbf{p})\right\rangle=:\left|\eta\left(\mathbf{p}, \Lambda_{j}\right)\right\rangle
$$

up to scalar multiplication for some $j=1, \ldots, 8$ and $\mathbf{p}=(p, q, r) \in \mathbb{R}_{+}^{3}$ satisfying the relation $p q^{-1} r^{-1}=u$, or equivalently

$$
p q^{-1}=u r, \quad q r=p u^{-1} \quad \text { or } \quad r p^{-1}=q^{-1} u^{-1} .
$$

For a given subset $\Sigma \subset \mathbb{R}_{+}^{3}$, we also define the set $F_{\Sigma}$ of product vectors by

$$
F_{\Sigma}=\left\{\left|\eta_{j}(\mathbf{p})\right\rangle: \mathbf{p} \in \Sigma, j=1,2, \ldots, 8\right\}
$$

We have seen that the set $P_{W}^{\mathrm{n}}$ of product vectors in $P_{W}$ without zero entries is given by $F_{S}$ with

$$
S=\left\{(p, q, r) \in \mathbb{R}_{+}^{3}: p q^{-1} r^{-1}=u\right\} .
$$

In short, we see that $P_{W}$ consists of product vectors in (1) and those in $F_{S}$.

We fix a point $\mathbf{p}$ in the surface $S$, and write $\varrho_{j}=:\left|\eta_{j}(\mathbf{p})\right\rangle\left\langle\eta_{j}(\mathbf{p})\right|$ for $j=1,2, \ldots, 8$. By the argument in Section III of [17], we see that the pure product states $\varrho_{1}, \varrho_{2}, \varrho_{3}$ and $\varrho_{4}$ share the common X-part, which is a separable $\mathbf{X}$-state of rank four. Furthermore, this $\mathrm{X}$-state is uniquely decomposed into the average of $\varrho_{1}, \varrho_{2}, \varrho_{3}$ and $\varrho_{4}$. See [11] for multi-qubit analogue. By a direct calculation, we have

$$
\varrho_{9}:=\frac{1}{4}\left(\varrho_{1}+\varrho_{2}+\varrho_{3}+\varrho_{4}\right)=X\left(a_{\mathbf{p}}, b_{\mathbf{p}},\left(\omega^{5}, \omega^{3}, \omega^{7}, \omega^{5}\right)\right),
$$

with the notations

$$
a_{\mathbf{p}}=\left(p^{2} u^{-1}, q^{2} u, r^{2} u, u\right), \quad b_{\mathbf{p}}=\left(p^{-2} u, q^{-2} u^{-1}, r^{-2} u^{-1}, u^{-1}\right) .
$$

On the other hand, the states $\varrho_{5}, \varrho_{6}, \varrho_{7}$ and $\varrho_{8}$ share the X-part

$$
\varrho_{10}:=\frac{1}{4}\left(\varrho_{5}+\varrho_{6}+\varrho_{7}+\varrho_{8}\right)=X\left(a_{\mathbf{p}}, b_{\mathbf{p}},\left(\omega^{3}, \omega^{5}, \omega, \omega^{3}\right)\right)
$$

which is again a rank four separable state with unique decomposition. Note that the average of all of them is given by

$$
\varrho_{\mathbf{p}}:=\frac{1}{2}\left(\varrho_{9}+\varrho_{10}\right)=\frac{1}{8} \sum_{j=1}^{8} \varrho_{j}=X\left(a_{\mathbf{p}}, b_{\mathbf{p}}, \frac{1}{\sqrt{2}}(-1,-1,+1,-1)\right) .
$$

Comparing the diagonal and anti-diagonal entries, we see that $\varrho_{\mathbf{p}}$ has the full rank eight. This shows that the eight product vectors $\left|\eta_{j}(\mathbf{p})\right\rangle$ with $k=1,2, \ldots 8$ make a basis of $\mathbb{C}^{2} \otimes \mathbb{C}^{2} \otimes \mathbb{C}^{2}$ for each fixed $\mathbf{p} \in S$.

We denote by $\Gamma_{A}, \Gamma_{B}$ ad $\Gamma_{C}$ the partial transposes with respect to the $A, B$ and $C$ parties, respectively. Then we have

$$
\begin{gathered}
X(a, b, c)^{\Gamma_{A}}=X\left(a, b,\left(\bar{c}_{4}, \bar{c}_{3}, \bar{c}_{2}, \bar{c}_{1}\right)\right), \\
X(a, b, c)^{\Gamma_{B}}=X\left(a, b,\left(c_{3}, c_{4}, c_{1}, c_{2}\right),\right. \\
X(a, b, c)^{\Gamma_{C}}=\underset{5}{5}\left(a, b,\left(c_{2}, c_{1}, c_{4}, c_{3}\right) .\right.
\end{gathered}
$$


Therefore, we see that all the partial transposes

$$
\begin{aligned}
& \left(\varrho_{\mathbf{p}}\right)^{\Gamma_{A}}=X\left(a_{\mathbf{p}}, b_{\mathbf{p}}, \frac{1}{\sqrt{2}}(-1,+1,-1,-1)\right), \\
& \left(\varrho_{\mathbf{p}}\right)^{\Gamma_{B}}=X\left(a_{\mathbf{p}}, b_{\mathbf{p}}, \frac{1}{\sqrt{2}}(+1,-1,-1,-1)\right), \\
& \left(\varrho_{\mathbf{p}}\right)^{\Gamma_{C}}=X\left(a_{\mathbf{p}}, b_{\mathbf{p}}, \frac{1}{\sqrt{2}}(-1,-1,-1,+1)\right)
\end{aligned}
$$

also have the full ranks. Hence, we have the following:

Proposition 2.1. The state $\varrho_{\mathbf{p}}$ is a boundary separable state with full ranks for every $\mathbf{p}$ on the surface $S$. Especially, eight product vectors $\left\{\left|\eta_{j}(\mathbf{p})^{\gamma}\right\rangle: j=1,2, \ldots, 8\right\}$ form a basis for each partial conjugate operation $\gamma$ and $\mathbf{p} \in S$.

\section{Construction}

We are going to construct entanglement witnesses whose dual faces have only finitely many extreme points. The main idea is to apply the bit-flip operators and partial transposes to the witness

$$
W=X\left(\left(0,0,0, \sqrt{8} u^{-1}\right),(0,0,0, \sqrt{8} u),(1,1,-1,1)\right),
$$

to get witnesses with the same anti-diagonals as $W$ but different diagonals from $W$. We will mix some of them to get entanglement witnesses whose dual faces have exactly ten extreme points. Recall the relation

$$
\left(W_{1}+W_{2}\right)^{\prime}=W_{1}^{\prime} \cap W_{2}^{\prime}
$$

for entanglement witnesses $W_{1}$ and $W_{2}$.

We denote by $\sigma$ the bit-flip operator $\left(\begin{array}{ll}0 & 1 \\ 1 & 0\end{array}\right)$ on $\mathbb{C}^{2}$, and define the operators on $\mathbb{C}^{2} \otimes \mathbb{C}^{2} \otimes \mathbb{C}^{2}$ by

$$
\sigma_{A}=\sigma \otimes I \otimes I, \quad \sigma_{B}=I \otimes \sigma \otimes I, \quad \sigma_{C}=I \otimes I \otimes \sigma,
$$

where $I$ denotes the identity operator. We note that

$$
\begin{aligned}
& \sigma_{A} X(a, b, c) \sigma_{A}=X\left(\left(b_{4}, b_{3}, b_{2}, b_{1}\right),\left(a_{4}, a_{3}, a_{2}, a_{1}\right),\left(\bar{c}_{4}, \bar{c}_{3}, \bar{c}_{2}, \bar{c}_{1}\right)\right), \\
& \sigma_{B} X(a, b, c) \sigma_{B}=X\left(\left(a_{3}, a_{4}, a_{1}, a_{2}\right),\left(b_{3}, b_{4}, b_{1}, b_{2}\right),\left(c_{3}, c_{4}, c_{1}, c_{2}\right)\right), \\
& \sigma_{C} X(a, b, c) \sigma_{C}=X\left(\left(a_{2}, a_{1}, a_{4}, a_{3}\right),\left(b_{2}, b_{1}, b_{4}, b_{3}\right),\left(c_{2}, c_{1}, c_{4}, c_{3}\right)\right) .
\end{aligned}
$$

Therefore, we have

$$
\begin{gathered}
W_{A}:=\sigma_{A} W^{\Gamma_{A}} \sigma_{A}=X\left((\sqrt{8} u, 0,0,0),\left(\sqrt{8} u^{-1}, 0,0,0\right),(1,1,-1,1)\right), \\
W_{B}:=\sigma_{B} W^{\Gamma_{B}} \sigma_{B}=X\left(\left(0, \sqrt{8} u^{-1}, 0,0\right),(0, \sqrt{8} u, 0,0),(1,1,-1,1)\right), \\
W_{C}:=\sigma_{C} W^{\Gamma_{C}} \sigma_{C}=X\left(\left(0,0, \sqrt{8} u^{-1}, 0\right),(0,0, \sqrt{8} u, 0),(1,1,-1,1)\right) . \\
6
\end{gathered}
$$


Now, we are looking for product vectors $|\xi\rangle=|x\rangle \otimes|y\rangle \otimes|z\rangle$ which are killed by the witness $W_{A}$. We note that

$$
\begin{aligned}
\left\langle W_{A}, \mid \xi\right\rangle\langle\xi \mid\rangle & =\left\langle\sigma_{A} W^{\Gamma_{A}} \sigma_{A}, \mid \xi\right\rangle\langle\xi \mid\rangle \\
& =\left\langle W^{\Gamma_{A}}, \mid \sigma_{A} \xi\right\rangle\left\langle\sigma_{A} \xi \mid\right\rangle \\
& =\left\langle W^{\Gamma_{A}}, \mid \sigma x\right\rangle|y\rangle|z\rangle\langle\sigma x|\langle y|\langle z \mid\rangle \\
& =\langle W, \mid \sigma \bar{x}\rangle|y\rangle|z\rangle\langle\sigma \bar{x}|\langle y|\langle z \mid\rangle .
\end{aligned}
$$

Motivated by this relation, we write

$$
\left|\xi_{A}\right\rangle=|\sigma \bar{x}\rangle \otimes|y\rangle \otimes|z\rangle, \quad\left|\xi_{B}\right\rangle=|x\rangle \otimes|\sigma \bar{y}\rangle \otimes|z\rangle, \quad\left|\xi_{C}\right\rangle=|x\rangle \otimes|y\rangle \otimes|\sigma \bar{z}\rangle,
$$

for a product vector $|\xi\rangle=|x\rangle \otimes|y\rangle \otimes|z\rangle$. Then we see that a product vector $|\xi\rangle$ satisfies the relation $|\xi\rangle \in P_{W_{A}}$ if and only if $\left|\xi_{A}\right\rangle \in P_{W}$, and similarly for $B$ and $C$ parties. Therefore, we see by (11) that the set $P_{W_{A}}^{z}, P_{W_{B}}^{\mathrm{z}}$ and $P_{W_{C}}^{\mathrm{z}}$ are given by

$$
\begin{aligned}
& P_{W_{A}}^{\mathrm{z}}:=E_{\square 01} \cup E_{\square 10} \cup E_{1 \square 0} \cup E_{0 \square 1} \cup E_{10 \square} \cup E_{01 \square}, \\
& P_{W_{B}}^{\mathrm{z}}:=E_{\square 11} \cup E_{\square 00} \cup E_{0 \square 0} \cup E_{1 \square 1} \cup E_{01 \square} \cup E_{10 \square}, \\
& P_{W_{C}}^{\mathrm{z}}:=E_{\square 00} \cup E_{\square 11} \cup E_{0 \square 1} \cup E_{1 \square 0} \cup E_{00 \square} \cup E_{11 \square} .
\end{aligned}
$$

Next, we look for product vectors in $P_{W_{A}}$ without zero entry. To do this, we first note the relation

$$
\sigma(p, \bar{\alpha})^{\mathrm{t}}=(\bar{\alpha}, p)^{\mathrm{t}}=p \bar{\alpha}\left(p^{-1}, \alpha\right)^{\mathrm{t}},
$$

from which we have

$$
\left|\eta_{j}(\mathbf{p})_{A}\right\rangle=\left|\eta_{j}\left(p^{-1}, q, r\right)\right\rangle, \quad j=1,2, \ldots, 8,
$$

up to scalar multiplications, and similar relations for $\left|\eta_{j}(\mathbf{p})_{B}\right\rangle$ and $\left|\eta_{j}(\mathbf{p})_{C}\right\rangle$;

$$
\begin{array}{rlrl}
\left|\eta_{j}(\mathbf{p})_{B}\right\rangle & =\left|\eta_{j}\left(p, q^{-1}, r\right)\right\rangle, & & j=1,2, \ldots, 8, \\
\left|\eta_{j}(\mathbf{p})_{C}\right\rangle & =\left|\eta_{j}\left(p, q, r^{-1}\right)\right\rangle, & j=1,2, \ldots, 8 .
\end{array}
$$

Now, we define surfaces

$$
\begin{aligned}
& S_{A}=\left\{(p, q, r):\left(p^{-1}, q, r\right) \in S\right\}=\left\{(p, q, r) \in \mathbb{R}_{+}^{3}: p^{-1} q^{-1} r^{-1}=u\right\}, \\
& S_{B}=\left\{(p, q, r):\left(p, q^{-1}, r\right) \in S\right\}=\left\{(p, q, r) \in \mathbb{R}_{+}^{3}: p q r^{-1}=u\right\}, \\
& S_{C}=\left\{(p, q, r):\left(p, q, r^{-1}\right) \in S\right\}=\left\{(p, q, r) \in \mathbb{R}_{+}^{3}: p q^{-1} r=u\right\} .
\end{aligned}
$$

Then we have

$$
P_{W_{A}}^{\mathrm{n}}=F_{S_{A}}, \quad P_{W_{B}}^{\mathrm{n}}=F_{S_{B}}, \quad P_{W_{C}}^{\mathrm{n}}=F_{S_{C}} .
$$

Now, we consider the intersections of two sets to get

$$
\begin{gathered}
P_{W} \cap P_{W_{A}}=E_{\square 01} \cup E_{\square 10} \cup F_{S \cap S_{A}}, \\
P_{W} \cap P_{W_{B}}=E_{0 \square 0} \cup E_{1 \square 1} \cup F_{S \cap S_{B}}, \\
P_{W} \cap P_{W_{C}}=E_{00 \square} \cup E_{11 \square} \cup F_{S \cap S_{C}} . \\
7
\end{gathered}
$$


We also have the following:

$$
\begin{aligned}
& P_{W_{A}} \cap P_{W_{B}}=E_{01 \square} \cup E_{10 \square} \cup F_{S_{A} \cap S_{B}}, \\
& P_{W_{B}} \cap P_{W_{C}}=E_{\square 00} \cup E_{\square 11} \cup F_{S_{B} \cap S_{C}}, \\
& P_{W_{C}} \cap P_{W_{A}}=E_{0 \square 1} \cup E_{1 \square 0} \cup F_{S_{C} \cap S_{A}} .
\end{aligned}
$$

We note that the sets $P_{W}, P_{W_{A}}, P_{W_{B}}$ and $P_{W_{C}}$ are parameterized by six spheres and eight two-dimensional surfaces, respectively. On the other hand, an intersection of two of them is parameterized by two spheres and eight curves. To be more precise, we note that

$$
S \cap S_{A}=\left\{\left(1, q, q^{-1} u^{-1}\right): 0<q<\infty\right\},
$$

and so we see that sides of the eight curves $F_{S \cap S_{A}}$ approach to the sphere given by $E_{\square 01}$ and the other sides approach to the sphere given by $E_{\square 10}$. The same description works for other intersections.

Now, we consider the intersection of three of them. For example, we have

$$
\begin{aligned}
P_{W} \cap P_{W_{A}} \cap P_{W_{B}} & =\left(P_{W} \cap P_{W_{A}}\right) \cap\left(P_{W} \cap P_{W_{B}}\right) \\
& =\left[\left(E_{\square 01} \cup E_{\square 10}\right) \cap\left(E_{0 \square 0} \cup E_{1 \square 1}\right)\right] \cup F_{S \cap S_{A} \cap S_{B}} .
\end{aligned}
$$

We see that $\left(E_{\square 01} \cup E_{\square 10}\right) \cap\left(E_{0 \square 0} \cup E_{1 \square 1}\right)$ consists of two product vectors $|1\rangle \otimes|0\rangle \otimes|1\rangle$ and $|0\rangle \otimes|1\rangle \otimes|0\rangle$. We also see that $(p, q, r) \in S \cap S_{A} \cap S_{B}$ if and only if the relation

$$
p q^{-1} r^{-1}=u, \quad p^{-1} q^{-1} r^{-1}=u, \quad p q r^{-1}=u
$$

holds if and only if $\mathbf{p}=\left(1,1, u^{-1}\right)$. Therefore, $F_{S \cap S_{A} \cap S_{B}}$ consists of eight product vectors. In short, the set $P_{W} \cap P_{W_{A}} \cap P_{W_{B}}$ consists of the following ten product vectors:

$$
P_{W} \cap P_{W_{A}} \cap P_{W_{B}}=\left\{|010\rangle,|101\rangle,\left|\eta_{j}\left(1,1, u^{-1}\right)\right\rangle(j=1,2, \ldots, 8)\right\} .
$$

The state $\varrho_{\mathbf{p}}$ in (2) with $\mathbf{p}=\left(1,1, u^{-1}\right)$ is given by

$$
\varrho_{\mathbf{p}}=X\left(\left(u^{-1}, u, u^{-1}, u\right),\left(u, u^{-1}, u, u^{-1}\right), \frac{1}{\sqrt{2}}(-1,-1,+1,-1)\right),
$$

and it is easily checked that $\left\langle W+W_{A}+W_{B}, \varrho_{\mathbf{p}}\right\rangle=0$.

By the exactly same way, we have

$$
\begin{aligned}
P_{W} \cap P_{W_{B}} \cap P_{W_{C}} & =\left\{|000\rangle,|111\rangle,\left|\eta_{j}(u, 1,1)\right\rangle(j=1,2, \ldots, 8)\right\}, \\
P_{W} \cap P_{W_{C}} \cap P_{W_{A}} & =\left\{|001\rangle,|110\rangle,\left|\eta_{j}\left(1, u^{-1}, 1\right)\right\rangle(j=1,2, \ldots, 8)\right\}, \\
P_{W_{A}} \cap P_{W_{B}} \cap P_{W_{C}} & =\left\{|011\rangle,|100\rangle,\left|\eta_{j}\left(u, u^{-1}, u^{-1}\right)\right\rangle(j=1,2, \ldots, 8)\right\} .
\end{aligned}
$$

We also have

$$
\begin{array}{r}
\varrho_{(u, 1,1)}=X\left((u, u, u, u),\left(u^{-1}, u^{-1}, u^{-1}, u^{-1}\right), \frac{1}{\sqrt{2}}(-1,-1,+1,-1)\right), \\
\varrho_{\left(1, u^{-1}, 1\right)}=X\left(\left(u^{-1}, u^{-1}, u, u\right),\left(u, u, u^{-1}, u^{-1}\right), \frac{1}{\sqrt{2}}(-1,-1,+1,-1)\right), \\
\varrho_{\left(u, u^{-1}, u^{-1}\right)}=X\left(\left(u, u^{-1}, u^{-1}, u\right),\left(u^{-1}, u, u, u^{-1}\right), \frac{1}{\sqrt{2}}(-1,-1,+1,-1)\right) .
\end{array}
$$

So far, we have seen that the dual face

$$
\left(W+W_{A}+W_{B}\right)_{8}^{\prime}=W^{\prime} \cap W_{A}^{\prime} \cap W_{B}^{\prime}
$$


of the witness $W+W_{A}+W_{B}$ has exactly ten pure product states. So, this face is the convex hull of the ten extreme points. It is well known that the convex hull of finitely many points on an affine manifold in a real vector space is a simplex if and only if they are linearly independent.

Theorem 3.1. Suppose that $F$ is the dual face of one of the entanglement witnesses

$$
W+W_{A}+W_{B}, \quad W+W_{B}+W_{C}, \quad W+W_{C}+W_{A}, \quad W_{A}+W_{B}+W_{C}
$$

Then we have the following:

(i) F is affinely isomorphic to the 9-dimensional simplex with ten extreme points,

(ii) every point of $F$ is a separable state with unique decomposition into the sum of pure product states,

(iii) every interior point of $F$ is a boundary separable state with full ranks, and has length ten.

Proof. We will prove for the entanglement $W+W_{A}+W_{B}$ only. For the statement (i), it remains to show that the ten pure product states arising from ten product vectors in (3) are linearly independent in the real vector space of all three qubit self-adjoint matrices. Write $\left|\eta_{j}\right\rangle=\left|\eta_{j}\left(1,1, u^{-1}\right)\right\rangle$ for $j=1,2, \ldots, 8$, and suppose that

$$
a|010\rangle\langle 010|+b| 101\rangle\left\langle 101\left|+\sum_{j=1}^{8} c_{j}\right| \eta_{j}\right\rangle\left\langle\eta_{j}\right|=0,
$$

with real numbers $a, b$ and $c_{j}$. Then we have

$$
0=a|010\rangle\langle 010 \mid 000\rangle+b|101\rangle\langle 101 \mid 000\rangle+\sum_{j=1}^{8} c_{j}\left|\eta_{j}\right\rangle\left\langle\eta_{j} \mid 000\right\rangle=\sum_{j=1}^{8} c_{j}\left|\eta_{j}\right\rangle\left\langle\eta_{j} \mid 000\right\rangle .
$$

We have already seen that eight vectors $\left\{\left|\eta_{j}\right\rangle: j=1, \ldots, 8\right\}$ form a basis, and so $c_{j}\left\langle\eta_{j} \mid 000\right\rangle=0$ for each $j=1,2, \ldots, 8$. Since $\left|\eta_{j}\right\rangle$ has no zero entry, we conclude that $\left\langle\eta_{j} \mid 000\right\rangle \neq 0$ and $c_{j}=0$ for each $j=1,2, \ldots, 8$. The second statement (ii) follows from (i). For (iii), we note that every interior point of $F$ must be expressed by

$$
a|010\rangle\langle 010|+b| 101\rangle\left\langle 101\left|+\sum_{j=1}^{8} c_{j}\right| \eta_{j}\right\rangle\left\langle\eta_{j}\right|
$$

in a unique way with $a>0, b>0$ and $c_{j}>0$ for each $j=1,2, \ldots, 8$. Therefore, the result follows from Proposition 2.1.

In the statement (iii) of Theorem [3.1, some boundary points of $F$ have still full ranks as well as interior points. In fact, every nine choice among ten product vectors spans the whole space $\mathbb{C}^{2} \otimes \mathbb{C}^{2} \otimes \mathbb{C}^{2}$. This is also the case when ten product vectors are replaced by their partial conjugates. If we take eight product vectors among ten, then they sometimes span the whole space, and sometimes do not span the whole space. We 
show this for ten product vectors

$$
\begin{aligned}
& \eta_{1}=\left(1,+\omega^{3}\right)^{\mathrm{t}} \otimes\left(1,+\omega^{1}\right)^{\mathrm{t}} \otimes\left(u^{-1},+\omega^{7}\right)^{\mathrm{t}}, \\
& \eta_{2}=\left(1,+\omega^{3}\right)^{\mathrm{t}} \otimes\left(1,-\omega^{1}\right)^{\mathrm{t}} \otimes\left(u^{-1},-\omega^{7}\right)^{\mathrm{t}}, \\
& \eta_{3}=\left(1,-\omega^{3}\right)^{\mathrm{t}} \otimes\left(1,+\omega^{1}\right)^{\mathrm{t}} \otimes\left(u^{-1},-\omega^{7}\right)^{\mathrm{t}}, \\
& \eta_{4}=\left(1,-\omega^{3}\right)^{\mathrm{t}} \otimes\left(1,-\omega^{1}\right)^{\mathrm{t}} \otimes\left(u^{-1},+\omega^{7}\right)^{\mathrm{t}}, \\
& \eta_{j}=\bar{\eta}_{j-4}, \quad j=5,6,7,8,
\end{aligned}
$$

in (3) together with $|010\rangle$ and $|101\rangle$.

Motivated by the relation $\left\langle(p, \alpha)^{t} \mid\left(p^{-1},-\alpha\right)^{t}\right\rangle=0$ for $p>0$ and $\alpha \in \mathbb{T}$, we define

$$
\begin{aligned}
& \zeta_{1}=\left(1,+\omega^{3}\right)^{\mathrm{t}} \otimes\left(1,-\omega^{1}\right)^{\mathrm{t}} \otimes\left(u,+\omega^{7}\right)^{\mathrm{t}}, \\
& \zeta_{2}=\left(1,+\omega^{3}\right)^{\mathrm{t}} \otimes\left(1,+\omega^{1}\right)^{\mathrm{t}} \otimes\left(u,-\omega^{7}\right)^{\mathrm{t}}, \\
& \zeta_{3}=\left(1,-\omega^{3}\right)^{\mathrm{t}} \otimes\left(1,-\omega^{1}\right)^{\mathrm{t}} \otimes\left(u,-\omega^{7}\right)^{\mathrm{t}}, \\
& \zeta_{4}=\left(1,-\omega^{3}\right)^{\mathrm{t}} \otimes\left(1,+\omega^{1}\right)^{\mathrm{t}} \otimes\left(u,+\omega^{7}\right)^{\mathrm{t}}, \\
& \zeta_{j}=\bar{\zeta}_{j-4}, \quad j=5,6,7,8 .
\end{aligned}
$$

Then the 'coefficient matrix' $L:=\left[\left\langle\zeta_{i} \mid \eta_{j}\right\rangle\right]_{i, j=1,2, \ldots, 8}$ of $\left\{\left|\eta_{j}\right\rangle: j=1,2, \ldots .8\right\}$ with respect to $\left\{\left|\zeta_{i}\right\rangle\right\}$ is given by

$$
L=\left(\begin{array}{cc}
0 & K \\
\bar{K} & 0
\end{array}\right) \in M_{2}\left(M_{4}\right), \quad \text { with } K=2 \sqrt{2} \omega^{3}\left(\begin{array}{ccc}
+ & - & - \\
- & + & - \\
- & - & - \\
- & - & -
\end{array}\right),
$$

where + and - denote +1 and -1 , respectively. Because $\operatorname{det} K \neq 0$, we see that both $\left\{\left|\eta_{j}\right\rangle\right\}$ and $\left\{\left|\zeta_{j}\right\rangle\right\}$ are linearly independent. If we take partial conjugates of $\left|\eta_{j}\right\rangle$ and $\left|\zeta_{i}\right\rangle$ in the $A, B$ and $C$ parties respectively, then $K / 2 \sqrt{2}$ is replaced by

$$
\omega^{-3}\left(\begin{array}{cccc}
- & + & - & - \\
+ & - & - & - \\
- & - & - & + \\
- & - & + & -
\end{array}\right), \quad \omega^{-3}\left(\begin{array}{cccc}
- & - & + & - \\
- & - & - & + \\
+ & - & - & - \\
- & + & - & -
\end{array}\right) \quad \text { and } \quad \omega^{-3}\left(\begin{array}{cccc}
- & - & - & + \\
- & - & + & - \\
- & + & - & - \\
+ & - & - & -
\end{array}\right)
$$

respectively. Therefore, all the partial conjugates of $\left\{\left|\eta_{j}\right\rangle\right\}$ are linearly independent. This gives another proof of Proposition 2.1.

If one of $\left|\eta_{j}\right\rangle$ is replaced by $|010\rangle$ and $|101\rangle$ respectively, then one column of $L$ is replaced by

$$
u\left(\omega^{3}, \omega^{7}, \omega^{3}, \omega^{7}, \omega^{5}, \omega^{1}, \omega^{5}, \omega^{1}\right)^{\mathrm{t}} \text { and }\left(\omega^{6}, \omega^{2}, \omega^{6}, \omega^{2}, \omega^{2}, \omega^{6}, \omega^{2}, \omega^{6}\right)^{\mathrm{t}},
$$

respectively. They are still non-singular, and the eight product vectors we have chosen are linearly independent. Especially, we see that every nine choice among ten product vectors spans the whole space $\mathbb{C}^{2} \otimes \mathbb{C}^{2} \otimes \mathbb{C}^{2}$. If all the $\left|\eta_{j}\right\rangle$ 's and $\left|\zeta_{i}\right\rangle$ 's are replaced by partial conjugate, then we have the same conclusion. Therefore, we have the following:

Proposition 3.2. Every nine choice among ten product vectors in

$$
P_{W} \cap P_{W_{A}} \cap P_{W_{B}}, \quad P_{W} \cap P_{W_{B}} \cap P_{W_{C}}, \quad P_{W} \cap P_{W_{C}} \cap P_{W_{A}} \quad \text { or } \quad P_{W_{A}} \cap P_{W_{B}} \cap P_{W_{C}}
$$


spans the whole space $\mathbb{C}^{2} \otimes \mathbb{C}^{2} \otimes \mathbb{C}^{2}$.

We give a geometric interpretation of Proposition 3.2. We begin with the real vector space of all self-adjoint three qubit matrices, and the 10-dimensional subspace generated by ten pure product states in Theorem 3.1. We consider the 9-dimensional affine manifold $H$ given by the condition of trace one. Then the dual face $F$ in Theorem 3.1 coincides with the convex set $\mathcal{S}_{H}=\mathcal{S} \cap H$ of all separable states on the affine manifold $H$. The boundary of $\mathcal{S}_{H}$ consists of maximal faces isomorphic to the 8dimensional simplex. We also consider the set $\mathcal{T}_{H}=\mathcal{T} \cap H$ of all PPT states on $H$. Proposition 3.2 tells us that the interior of these maximal faces are contained in the interior of $\mathcal{T}_{H}$. Take an interior point $\varrho_{0}$ of $\mathcal{S}_{H}$ and an interior point $\varrho_{1}$ of a maximal face of $\mathcal{S}_{H}$. Take also the line segment $\varrho_{t}=(1-t) \varrho_{0}+t \varrho_{1}$ from $\varrho_{0}$ to $\varrho_{1}$. Because $\varrho_{1}$ is an interior point of $\mathcal{T}_{H}$, there exists $t>1$ so that $\varrho_{t} \in \mathcal{T}_{H}$, which is a PPT entangled state. In this way, we have bunch of PPT entanglement, as in [14, 15]. Note that $\varrho_{1}$ is a separable state of length 9 with full ranks and unique decomposition.

The same argument may hold even if we take $\varrho_{1}$ on the boundary of a maximal face. In fact, if we take the face generated by eight pure product states corresponding to $\left|\eta_{j}\right\rangle$

with $j=1,2, \ldots, 8$, then this face is on the boundary of a maximal face, but still in the interior of $\mathcal{T}_{H}$ by Proposition 2.1. In this case, $\varrho_{1}$ is a separable state of length 8 with full ranks and unique decomposition. But, this is not the case in general. Suppose that two of $\left|\eta_{1}\right\rangle, \ldots,\left|\eta_{4}\right\rangle$ are replaced by $|010\rangle,|101\rangle$ to get the eight product vectors. Then we see that the matrix $\bar{K}$ should be replaced by a matrix with two columns of same direction, while the lower-right corner is still zero. This implies that the coefficient matrix with respect to $\left\{\left|\zeta_{j}\right\rangle\right\}$ is singular, and those eight product vectors are linearly dependent. The convex hull of the corresponding eight pure product states generate a face isomorphic to the 7-dimensional simplex which is contained in the boundary of $\mathcal{T}_{H}$, and so the line segment from $\varrho_{0}$ to an interior point of this face cannot be extended in $\mathcal{T}_{H}$. If we replace one of $\left|\eta_{1}\right\rangle, \ldots,\left|\eta_{4}\right\rangle$ by $|010\rangle$ and replace one of $\left|\eta_{5}\right\rangle, \ldots,\left|\eta_{8}\right\rangle$ by $|101\rangle$ then we can see that the resulting coefficient matrix is non-singular.

\section{REFERENCES}

[1] E. Alfsen and F. Shultz, Unique decompositions, faces, and automorphisms of separable states, J. Math. Phys. 51 (2010), 052201.

[2] E. Alfsen and F. Shultz, Finding decompositions of a class of separable states, Linear Alg. Appl. 437 (2012), 2613-2629.

[3] L. Chen and D. Ž. Djoković, Qubit-qudit states with positive partial transpose, Phys. Rev. A 86 (2012), 062332.

[4] L. Chen and D. Ž. Djoković, Dimensions, lengths and separability in finite-dimensional quantum systems, J. Math. Phys. 54, (2013), 022201.

[5] L. Chen and D. Ž. Djoković, Properties and construction of extreme bipartite states having positive partial transpose, Commun. Math. Phys. 323 (2013), 241-284.

[6] L. Chen and D. Ž. Djoković, Boundary of the set of separable states, Proc. R. Soc. A 471 (2015), 20150102. 
[7] L. Chen and D. Ž. Djoković, Dimension formula for induced maximal faces of separable states and genuine entanglement, Quantum Information Processing 14 (2015), 3335-3350.

[8] L. Chen and D. Ž. Djoković, Length filtration of the separable states, Proc. R. Soc. A 472 (2016), DOI: $10.1098 /$ rspa.2016.0350.

[9] S. M. Cohen, Sums of product operators that remain product operators, preprint. arXive 1210.0644

[10] D. P. DiVincenzo, B. M. Terhal, and A. V. Thapliyal, Optimal decompositions of barely separable states, J. Mod. Opt. 47 (2000), 277-385.

[11] K.-C. Ha, K. H. Han and S.-H, Kye, Separability of multi-qubit states in terms of diagonal and anti-diagonal entries, preprint. arXiv 1803.00175

[12] K.-C. Ha and S.-H. Kye, Exposedness of Choi type entanglement witnesses and applications to lengths of separable states, Open Syst. Inf. Dyn. 20 (2013), 1350012.

[13] K.-C. Ha and S.-H. Kye, Geometry for separable states and construction of entangled states with positive partial transposes, Phys. Rev. A 88 (2013), 024302.

[14] K.-C. Ha and S.-H. Kye, Separable states with unique decompositions, Commun. Math. Phys. 328 (2014), 131-153.

[15] K.-C. Ha and S.-H. Kye, Multi-partite separable states with unique decompositions and construction of three qubit entanglement with positive partial transpose, J. Phys. A: Math. Theor. 48 (2015), 045303.

[16] K. H. Han and S.-H, Kye, Construction of multi-qubit optimal genuine entanglement witnesses, J. Phys. A: Math. Theor. 49 (2016), 175303.

[17] K. H. Han and S.-H, Kye, The role of phases in detecting three qubit entanglement, J. Math. Phys. 58 (2017), 102201.

[18] P. Horodecki, Separability criterion and inseparable mixed states with positive partial transposition, Phys. Lett. A 232 (1997), 333-339.

[19] K. A. Kirkpatrick, Uniqueness of a convex sum of products of projectors, J. Math. Phys. 43 (2002), 684-686.

[20] Y.-H. Kiem, S.-H. Kye and J. Na, Product vectors in the ranges of multi-partite states with positive partial transposes and permanents of matrices, Commun. Math. Phys. 338 (2015), 621-639.

[21] S.-H. Kye, Three-qubit entanglement witnesses with the full spanning properties J. Phys. A: Math. Theor. 48 (2015), 235303.

[22] S.-H. Kye, Indecomposable exposed positive bi-linear maps between two by two matrices, Acta Math. Viet. (to appear), arXiv 1709.06705.

[23] R. Lockhart, Optomal ensemble length of mixed separable states, J. Math. Phys. 41 (2000), 67666771.

[24] A. Sanpera, R. Tarrach and G. Vidal, Local description of quantum inseparability, Phys. Lett. A 58 (1998), 826-830.

[25] L. Skowronek, Dualities and positivity in the study of quantum entanglement, Intern. J. Quantum Inform. 8 (2010), 721-754.

[26] A. Uhlmann, Entropy and optimal decompositions of states relative to a maximal commutative subalgebra, Open Sys. Inf. Dyn. 5 (1998) 209-227.

[27] J. Walgate and A. J. Scott, Generic local distinguishability and completely entangled subspaces, J. Phys. A 41 (2008), 375305.

[28] W. K. Wootters, entanglement of formation of an arbitrary state of two qubits, Phys. Rev. Lett. 80 (1998), 2245-2248.

Department of Mathematics and Institute of Mathematics, Seoul National UniVERSity, SEOUl 151-742, Korea

E-mail address: kye at snu.ac.kr 